\title{
Magnetic properties of ZnO Nanoparticles
}

M. A. Garcia ${ }^{1,2 *}$, J. M. Merino ${ }^{1,3}$, E. Fernández Pinél1,2, A. Quesada ${ }^{1,2}$, J. De la Venta ${ }^{1,2}$, M. L. Ruiz Gonzalez ${ }^{3}$, G. Castro ${ }^{4}$, P. Crespo ${ }^{1,2}$, J. Llopis ${ }^{2}$, J. M. González-Calbet ${ }^{1,3}$, A. Hernando ${ }^{1,2}$.

${ }^{1}$ Instituto de Magnetismo Aplicado (UCM-RENFE-CSIC), P.O. Box 155, 28230 Las Rozas, Madrid, Spain.

${ }^{2}$ Departamento de Física de Materiales, UCM, 28040 Madrid, Spain ${ }^{3}$ Departamento de Química Inorgánica I, UCM, 28040 Madrid, Spain

${ }^{4}$ SpLine, Spanish CRG beamline at the ESRF, B.P. 220, F-38043 Grenoble, France and ICMM-CSIC Cantoblanco, E-28049 Madrid, Spain

Corresponding autor: e-mail: miguelag@adif.es

\begin{abstract}
In this work it is experimentally shown that capping $\mathrm{ZnO}$ nanoparticles with organic molecules leads to the appearance of magnetism at room temperature. The bonds between the molecules and the $\mathrm{Zn}$ atoms at the nanoparticle surface alter its electronic structure (as XANES and photoluminescence spectra demonstrate) arising magnetic moments with values that depend on the nature of the molecule. This result points out the possibility to observe magnetism at nanoscale in semiconductors without typical magnetic atoms (transition metals and rare earths).
\end{abstract}




\section{Text}

Diluted Magnetic Semiconductors (DMS), consisting on semiconductor matrices containing a small amount of magnetic impurities are among the most interesting new magnetic materials in view of their potential applications for spintronics $[1,2]$. The main challenge for this kind of materials is to present a Curie Temperature $\left(\mathrm{T}_{\mathrm{C}}\right)$ above $300 \mathrm{~K}$ in order to be useful for technological applications. Despite some initial promising results on $\mathrm{Mn}: \mathrm{ZnO}$ [3], it is not clear if DMS can exhibit this required high temperature magnetism. For most of the experimental results doubts arose about the real origin of magnetism $[4,5,6]$. The most recent and outstanding works on this field showed that the magnetic properties are not exclusively related to the presence of the magnetic ions but strongly determined by the defects in the host matrix $[7,8,9,10,11,12]$. For instance, Kittisltved et al $[7,8]$ showed that $\mathrm{Mn}: \mathrm{ZnO}$ nanoparticles and thin films show room temperature (RT) ferromagnetism when doped p-type defects, while other capping that introduce n-type defects leads to no RT ferromagnetism. On the contrary, for Co:ZnO films the n-type defects favours the appearance of RT ferromagnetism while p-type defects yield to no RT ferromagnetism. Rubi et al [9] found similar results for Co and Mn doped $\mathrm{ZnO}$ powder samples. Moreover, Coey et al [10] demonstrated that doping $\mathrm{ZnO}$ thin films with $3 \mathrm{~d}$ non magnetic ions (as Ti or V) also leads to RT ferromagnetic behaviour and for insulating $\mathrm{HfO}_{2}$ the effect appear even without doping [11]. Those results point on the alteration of the electronic structure of the semiconductor (induced by both, the presence of the magnetic impurity and the defects) as responsible of the origin of the observed magnetism.

Actually, it was recently found that an alteration of the electronic structure of $\mathrm{Au}$ nanoparticles and thin films produced by capping them with organic molecules also lead to the appearance of RT ferromagnetism despite the diamagnetic character of bulk Au $[13,14,15]$. When thiols are chemisorbed on $\mathrm{Au}$ surface, there is a charge transfer from the Au to the $\mathrm{S}$ [16] generating holes at the $5 \mathrm{~d}$ orbital of $\mathrm{Au}$ involved in the bond, initially full, arising a magnetic moment. These moments results strongly fixed along the bond direction and the system exhibits giant anisotropy. Thus, the ferromagnetic 
behaviour is observed even at RT. For thiol capped thin films the magnetization curves are fairly different upon applying field parallel or perpendicular to the surface (hence confirming the huge anisotropy) and the magnetic moments are giant $[14,15]$. Similar features (giant magnetic moments and huge anisotropy) have been observed for DMS thin films $[10,11,17]$ suggesting a possible common origin of both effects. In this work we experimentally show that capping $\mathrm{ZnO}$ NPs with a variety of organic molecules modifies its electronic structure arising ferromagnetic-like behaviour up to 300 K. Therefore, this method opens a new way to obtain the desired magnetic semiconductors without using any type of magnetic atoms and avoiding the superparamagnetic limit.

ZnO NPs were prepared by sol-gel following the method described in $[7,18,19]$ and subsequently capped with three different organic molecules: Tryoctylphosphine (named after here TOPO), dodecylamine (AMINE) and dodecanethiol (THIOL), which bond to the particle surface through an $\mathrm{O}, \mathrm{N}$ and $\mathrm{S}$ atom, respectively. Zinc acetate dihydrate $\left(\mathrm{Zn}(\mathrm{Ac})_{2}, \mathrm{Zn}\left(\mathrm{CH}_{3} \mathrm{CO}_{2}\right)_{2} \cdot 2 \mathrm{H}_{2} \mathrm{O}\right.$, $98 \%$, Sigma-Aldrich, $\leq 5 \mathrm{ppm}$ of $\mathrm{Fe}$ impurities), tetramethylammonium hydroxide pentahydrate (TMAH, $\mathrm{N}\left(\mathrm{CH}_{3}\right)_{4} \mathrm{OH}, 97 \%$, Sigma-Aldrich), dimethyl sulfoxide (DMSO, $\left(\mathrm{CH}_{3}\right)_{2} \mathrm{SO}$, Sigma-Aldrich), dodecylamine $\left(\mathrm{C}_{12} \mathrm{H}_{25} \mathrm{NH}_{2}, \geq 98 \%\right.$, Merck), dodecanethiol $\left(\mathrm{C}_{12} \mathrm{H}_{25} \mathrm{SH}, 98 \%\right.$, Sigma-Aldrich), tryoctylphospine oxide (TOPO, $\left(\mathrm{C}_{8} \mathrm{H}_{17}\right)_{3} \mathrm{PO}, 90 \%$ technical grade, Sigma-Aldrich) and absolute ethanol $\left(\mathrm{CH}_{3} \mathrm{CH}_{2} \mathrm{OH}\right.$, Panreac) were used as received. $\mathrm{ZnO}$ NPs were prepared by a solgel method. $\mathrm{Zn}(\mathrm{Ac})_{2}(5 \mathrm{mmol})$ was dissolved in DMSO and the solution was heated and kept at $60{ }^{\circ} \mathrm{C}$ under stirring. Then a solution of TMAH (7.5 mmol) in ethanol was added dropwise. After that, AMINE, THIOL and TOPO capped nanocrystals were precipitaded adding dodecylamine $(7.5 \mathrm{mmol})$, dodecanethiol $(7.5 \mathrm{mmol})$ and TOPO $(7.5 \mathrm{mmol})$ solutions in heated ethanol respectively to the precursor solution. The products thus obtained were filtered and washed several times with heated ethanol. The recovered powders were allowed to dry at room temperature.

Structural characterization, by means of X Ray Diffraction (XRD) and Transmission Electron Microscopy (TEM), evidences, for the three cases, the formation of hexagonal $\mathrm{ZnO}$ nanoparticles (wurtzite structural type) with average size around $10 \mathrm{~nm}$ as figure 1 illustrates. Figure $1 \mathrm{~b}$ presents a low magnification image, characteristic of the AMINE sample, where the NPs 
average size can be estimated. Figure 1c corresponds to a HREM (High Resolution Electron Microscopy) image of one of these particles, in which atomic distances and the corresponding FFT (figure 1d) confirms the hexagonal lattice. Similar results are obtained for THIOL and TOPO samples. EDS (Energy Dispersive Spectroscopy) analysis is in agreement with the presence of $\mathrm{ZnO}$. Signals corresponding to impurity phases have not been detected.
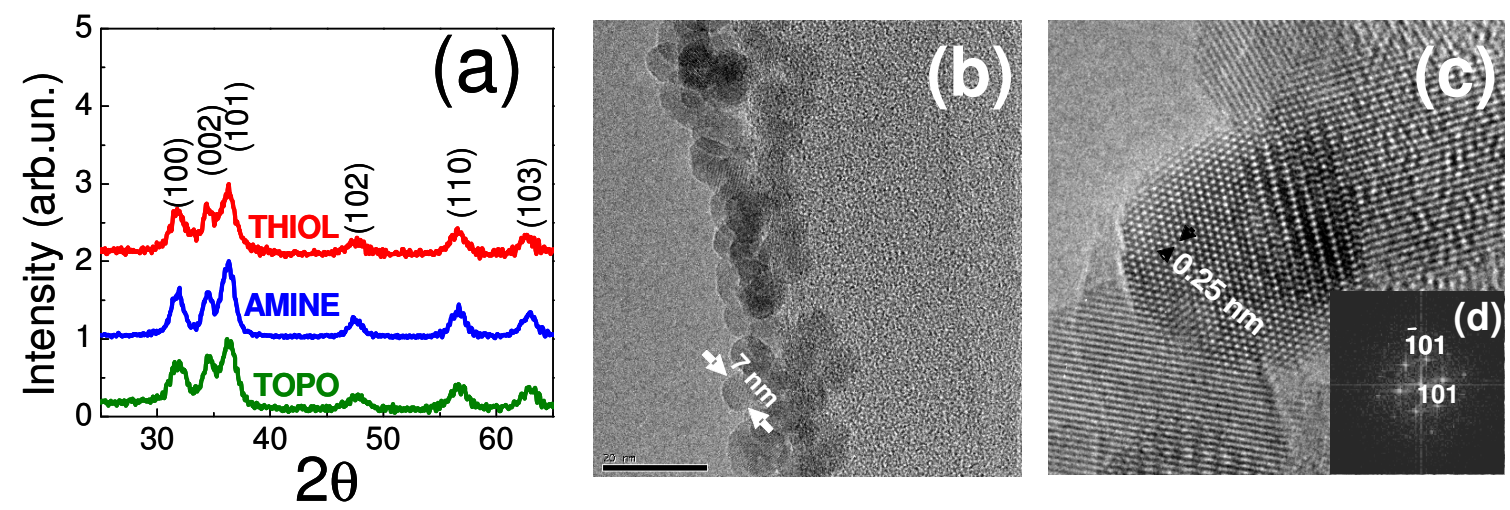

Figure 1. (Color on-line) (a) X-Ray diffraction patterns of the AMINE, THIOL and TOPO samples; diffraction maxima (labelled) are indexed on the basis of a $\mathrm{ZnO}$ wurtzite type unit cell. (b) Low magnification image of the AMINE sample, showing the size distribution. (c) HREM image along [010] zone axis and (d) corresponding FFT in agreement to the wurtzite unit cell.

Figure 2 show the XANES spectra at the Zn K-edge measured at RT in the SPLINE (BM25) beamline at ESRF. The spectra are clearly different for the three capping molecules. The $\mathrm{Zn} \mathrm{K}$-edge, corresponding to the transition $\mathrm{Zn}$ $1 \mathrm{~s} \rightarrow 4 \mathrm{p}$, has been shown to be more sensible to the $\mathrm{Zn}$ chemical bonding than $\mathrm{L}$ edges [20]. Although $4 \mathrm{p}$ level of $\mathrm{Zn}$ isolated atoms is empty, chemical bonding with different chemical species leads to hybridization, resulting states with a different degree of occupation depending on the features of the bond. In particular, a larger intensity at the first maximum is associated with a larger charge transfer between the $\mathrm{Zn}$ atoms and the surrounding atoms, that can be due to an increase of the coordination number [21] or changes in the electronegativity of these surrounding atoms. Hence, variations in intensity at the first maximum indicate different degree of occupation for the outer orbital of $\mathrm{Zn}$ atoms (including the hybridized states) for the three samples. 


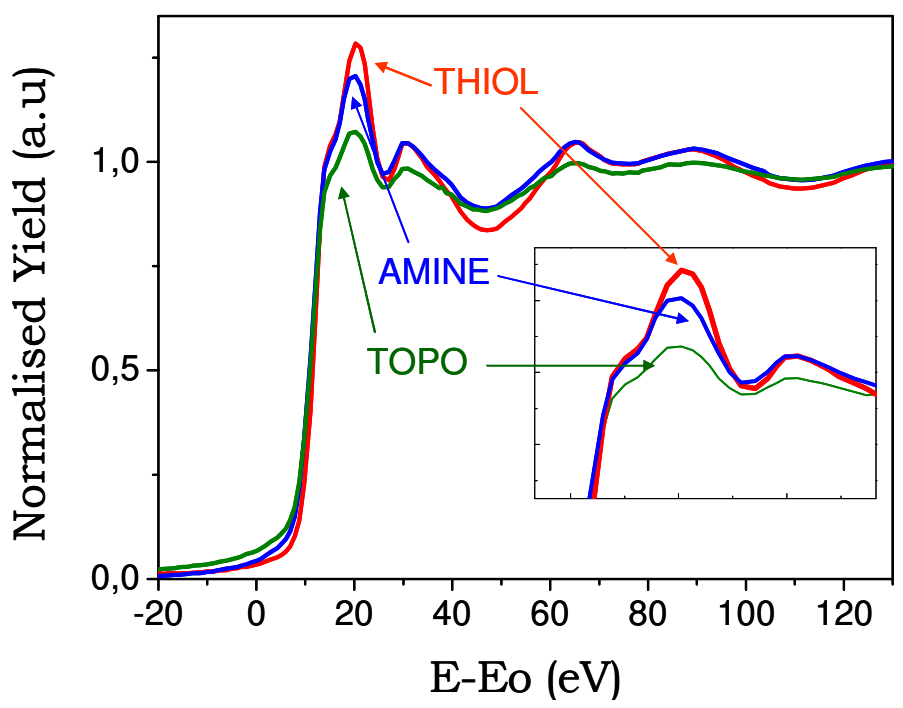

Figure 2. (Color on-line) XANES spectra at the $\mathrm{Zn}$ K-edge for the $\mathrm{ZnO}$ NPs capped with different molecules. Inset show a detail of the white line region.

The photoluminescence (PL) spectra of the $\mathrm{ZnO}$ NPs are presented in figure 3. As figure $3 a$ describes, upon excitation with UV light, electrons are pumped from the valence band (VB) to the conduction band (CB). Those electrons rapidly decay to the bottom of the $\mathrm{CB}$ via non-radiative processes. At this stage, there are several paths to return to the ground state, being the main ones: (a) Creation of an exciton and its subsequent annihilation emitting the excess of energy as a photon with an energy about that of the $\mathrm{ZnO}$ gap (3.4 eV) (b) Non-radiative transition to an intermediate level created by a defect and subsequent radiative decay emitting $2.3 \mathrm{eV}$ photons. Although this emission has been reported many times, the nature of the defect is still a matter of discussion, being the oxygen vacancies the most likely candidate [22]. Recently, Shalish et al [23] demonstrated that this luminescence arise form the surface of the $\mathrm{ZnO}$ while Norberg and Gamelin established it is directly correlated with the surface hydroxide concentration [24]. Thus, the study of this emission is a suitable tool to investigate the modification in the surface electronic structure induced by capping the NP with different molecules. (c) Surface states can create intermediate levels in the gap that allow the nonradiative decay of the excited electron to the VB $[25,26]$.

The $2.3 \mathrm{eV}$ emission (corresponding to photons with $550 \mathrm{~nm}$ wavelength) is clearly observed for the TOPO sample, being weaker for the AMINE and absent of the spectrum for the THIOL sample, as figure $3 \mathrm{~b}$ shows. The strong 
dependence of the PL visible emission with the type of capping molecule is in agreement with the surface origin [23,24], as the molecules can alter only the electronic structure close to the surface. These results can be understood assuming that the kind of molecule controls the number of deep-level recombination centers or, that it induces new surface states that provide alternative non-radiative decay paths, and hence quenching the PL emission [25]. In both cases, it is inferred that the capping molecule alters the electronic structure of the NPs surface, modifying the energy levels.

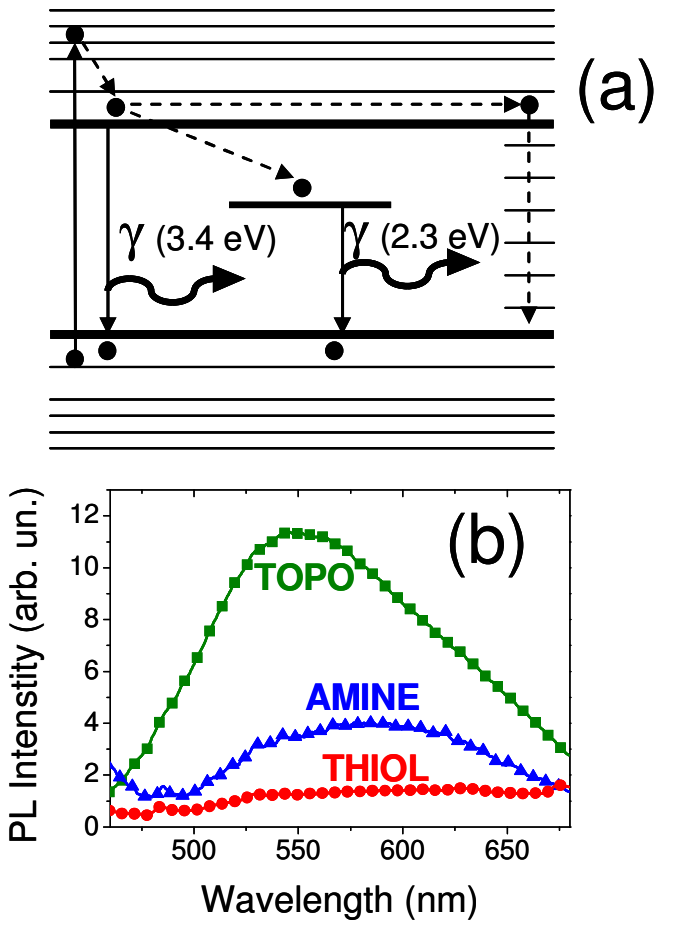

Figure 3. (a) Scheme of the PL process for $\mathrm{ZnO}$ as described in the text (b) PL spectra from the three samples at $300 \mathrm{~K}$ upon excitation with $385 \mathrm{~nm}$ light.

Figures 4 show the magnetization curves of the samples at $300 \mathrm{~K}$, exhibiting diamagnetic character as bulk $\mathrm{ZnO}$ does. However, for the AMINE and THIOL capped NPs there is a ferromagnetic-like contribution that can be clearly observed after subtracting the diamagnetic background (Figure 4b). Identical results were found at $5 \mathrm{~K}$. Actually the magnetization resulted thermally independent in the range $5-300 \mathrm{~K}$ (see inset). For an average nanoparticle size of $10 \mathrm{~nm}$, the magnetic moment per surface atom resulted $2 \cdot 10^{-3} \mu_{\mathrm{B}}$ and $0.5 \cdot 10^{-3} \mu_{\mathrm{B}}$ for THIOL and AMINE sample respectively. These values are calculated assuming that no rests of the precursors are present in the sample; 
they represent therefore a lower limit. Chemical analysis provided by the precursors suppliers showed that possible traces, lower than $0.5 \mathrm{ppm}$, can not account for the magnetic moment experimentally measured. Moreover, we measured the magnetization curves for the precursors used in the NP synthesis to check any contamination in the measuring procedure and we found no magnetic signal up to the detection limit of our equipment. Thus, possible contribution of magnetic impurities should be two orders of magnitude below that measured for THIOLS and one order for AMINE.
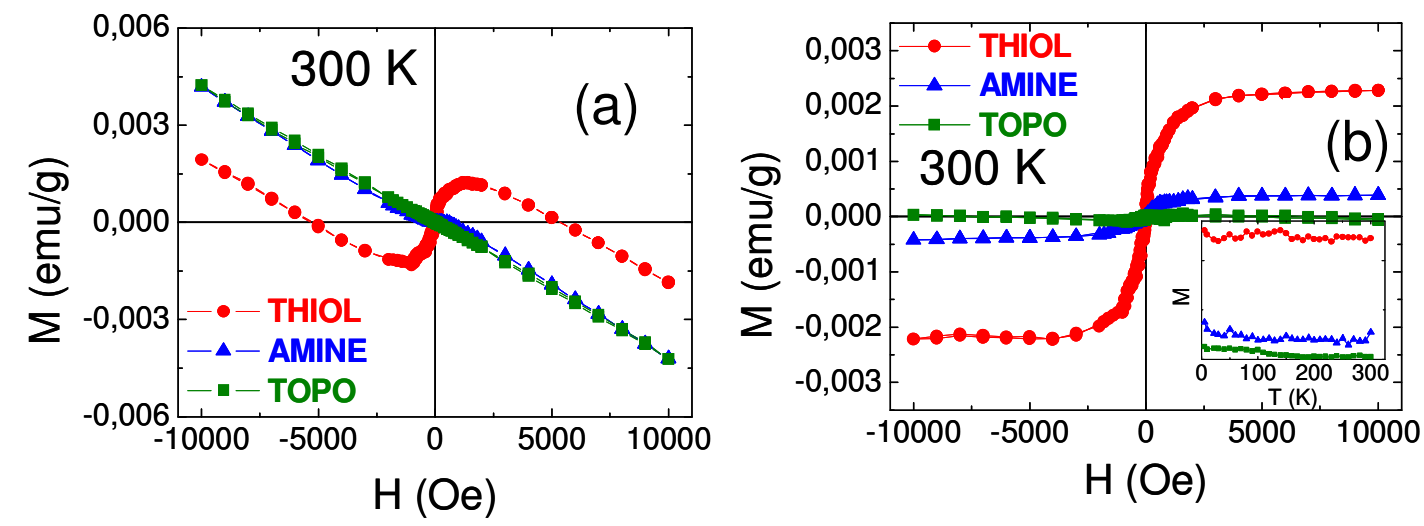

Figure 4. (a) Hysteresis loops from ZnO NPs capped with different molecules. (b) The loops alter subtracting the diamagnetic/paramagnetic background. Inset shows the thermal dependence of the under an applied field of 500 Oe after subtracting the corresponding constant diamagnetic background for clarity.

It is worthy noting that structural analysis of the NPs (performed by XRD, TEM and HREM) showed about identical structure irrespective of the capping molecule but those measurements probing the electronic structure (XANES, PL and magnetic properties) confirmed that these are strongly dependent on the capping molecule. Moreover, there is a perfect correlation in the evolution of these measured properties with the capping molecule: THIOL sample shows the largest alteration of the electronic structure as evidenced by the highest XANES absorption, the absence of PL emission (totally quenched by appearance the new surface states) and the largest magnetic moments (despite the diamagnetic character of $\mathrm{ZnO}$ ). On the contrary, the TOPO sample presents the electronic configuration more similar to bulk $\mathrm{ZnO}$ as confirms the smallest XANES absorption (less charge transfer), the most intense PL 
emission typical of bulk $\mathrm{ZnO}$ and diamagnetic behaviour. AMINE sample present in the three cases an intermediate result. All this results together point out that the capping molecule induces a modification of the electronic structure of the nanoparticles that is in the origin of the room temperature ferromagnetism of those samples.

In view of those results, the confusing and apparently irreconcilable results about DMS can be understood assuming that that their magnetic properties are originated by two sources: (i) The magnetism arising by the magnetic moments of the $3 \mathrm{~d}$ impurities that, when isolated, are expected to behave as paramagnetic and rapidly decrease with temperature, and (ii) an additional contribution originated by the alteration of the electronic structure of the semiconductor induced by defects ( $\mathrm{p}$ or $\mathrm{n}$ type) that is thermally independent up to $300 \mathrm{~K}$.

Previous experiments on DMS demonstrates that when both contributions are present they are coupled [7,8]. Actually, the incorporation of magnetic impurities into the semiconductor matrix, besides the presence of its magnetic moment, represents an alteration of the semiconductor energy levels. Thus, the final electronic configuration will be determined by both non-magnetic defects and magnetic impurities, explaining why the RT ferromagnetism depends also on the presence of the magnetic ion. The observation of RT ferromagnetism in DMS without defects can be explained as due to the alteration of the changes in the energy levels induced by the presence of the impurity. Moreover, the ferromagnetism observed in semiconductors doped with non-magnetic ions can be also explained within this frame. What we confirmed here is that the magnetism due to the alteration of the semiconductor electronic configuration can be present in absence of the magnetic ions and that, for nanoparticles, capping with organic molecules may induce such an alteration of the electronic structure.

In a recent work, Kittilstved and Gamelin found no RT ferromagnetism in AMINE capped $\mathrm{ZnO}$ NPs in disagreement with the result presented here [7]. It is evident that not any alteration of the electronic structure of $\mathrm{ZnO}$ will arise the ferromagnetism but only under certain circumstances. Actually, we found that slight modifications in the preparation conditions and size of thiol-capped Au NPs lead to important variations in their magnetic properties [13], and for DMS small differences in the preparation methods also lead to apparently 
irreconciliable results, suggesting that slight variation of the electronic structure can lead to large variation of the magnetic properties. Thus, it is possible that the different preparation method used by Kittilstved and Gamelin, yield reduced values of magnetization for their samples that make the magnetic moments undetectable (note that the magnetization value we measured for AMINE is fairly small and close to the detection limit of the SQUID). Then, for NPs capped with AMINE and doped with Mn atoms the electronic structure (modified both by the presence of $\mathrm{Mn}$ and the capping molecules) arise the thermally independent magnetization that is superimposed to that due to the magnetic moments of Mn atoms that decreases fast with temperature (Figure 1 in reference [7]).

In summary, we have experimentally shown that absorption of certain organic molecules onto semiconductor nanoparticles modifies its electronic structure and gives rise to a ferromagnetic-like behaviour at room temperature even in absence of magnetic ions. Identification of the particular electronic configuration that originates this magnetism is a amazing challenge that would open a world of possibilities for the use of these materials. 
Acknowledgements: The authors acknowledge to Prof. D. R. Gamelin for a critic reading of the manuscript and his valuable comments. This work has been supported by the Spanish Ministry of Education and Science (project NAN2004-09125-C07-05). 


\section{References}

[1] H. Ohno. Science 281, 951 (1998).

[2] T. Dietl, ,H. Ohno, F. Matsukura, J. Cibert, D. Ferrand, Science 287, 1019 (2000).

[3] P. Sharma et al. Nature Mater. 2, 673-677 (2003).

[4] D. C. Kundaliya, Nature Mater. 3, 709-714 (2004).

[5] G. Lawes, A. S. Risbud, A. P. Ramirez, R. Seshadri, Phys. Rev. B 71, 045201 (2005).

[6] M. A. García, M. A. et al. Phys. Rev. Lett. 94, 217206 (2005).

[7] K. R. Kittilstved, D. R. Gamelin, J. Am. Chem. Soc. 127, 5292 (2005).

[8] K. R. Kittilstved, N. S. Norberg, D. R. Gamelin, Phys. Rev. Lett. 94, 147209 (2005).

[9] D. Rubi et al, cond-mat/0608014. D. Rubi, PhD thesis Universitat . Autonoma de Barcelona, 2006.

[10] J. M. D. Coey, M. Venkatesan, C. B. Fitzgerald, Nature Mat. 4, 173 (2006).

[11] M. Venkatesan, C. B. Fitzgerald, J. M. D. Coey Nature 430, 630 (2004).

[12] K. R. Kittilstved, W. L Liu, D. R. Gamelin, Nature Mat. 5, 291 (2006).

[13] P. Crespo et al Phys. Rev. Lett. 93, 087204 (2004).

[14] I. Carmeli, G. Leitus, R. Naaman, S. Reich, Z. Vager. J. Chem. Phys. 18, 10372 (2003).

[15] A. Hernando et al, Phys. Rev. B 74, 052403 (2006).

[16] P. Zhang, T. K. Sham, Phys. Rev. Lett. 90, 245502 (2003).

[17] S. Dahr, L. Perez, O. Brandt, A.Trampert, K. H. Ploog, Phys. Rev. B 72 245203 (2005).

[18] N. S. Norberg et al, J. Am. Chem. Soc., 126, 3987 (2006).

[19]D. A. Schwartz et al J. Am. Chem. Soc., 125, 13205 (2003)

[20] T. Mizoguchi eta al, Phys. Rev. B, 70045103 (2004).

[21] C Hennig, K. H. Hallmeier, G. Zahn, F. Tschwatschal, H. Hennig, Inorg. Chem. 38, 38 (1999).

[22] A. Van Dijken, E. A. Meulemkamp, D. Vanmaekelbergh, A. Meijerink, J. Phys. Chem. B 104, 1715 (2000).

[23] I. Shalish, H. Temkin V. Narayanamurti Phys. Rev. B 69, 245401 (2004).

[24] N. S. Norberg, D. R. Gamelin, J. Phys. Chem. B 109, 20810 (2005).

[25] K. Borgohain, S. Mahamuni Semicond. Sci. Technol. 13, 1154 (1998).

[26] L. Guo et al, Appl. Phys. Lett. 76, 2901 (2000). 